\title{
INFORMAÇÕES SOBRE GESTÃO DE RISCOS NAS IANS DAS EMPRESAS LISTADAS NO NOVO MERCADO DA BOVESPA
}

\section{DATA ON RISK MANAGEMENT ON THE IAN (ANUAL INFORMATIONS) OF THE COMPANIES LISTED IN BOVESPA'S NEW MARKET}

\section{INFORMACIONES SOBRE GESTIÓN DE RIESGOS EN LAS IAN DE LAS EMPRESAS LISTADAS EN EL NUEVO MERCADO DE LA BOVESPA}

\author{
FRANCISCO CARLOS FERNANDES \\ Doutor em Controladoria e Contabilidade pela FEA/USP. \\ Professor do Programa de Pós-Graduação em Ciências Contábeis \\ da Universidade Regional de Blumenau - FURB. \\ fernandes.francisco@uol.com.br \\ MARCELO SILVA \\ Mestrando em Ciências Contábeis \\ na Universidade Regional de Blumenau - FURB. \\ smmarcelo@terra.com.br \\ FABIANO TERMUS DOS SANTOS \\ Mestrando em Ciências Contábeis \\ na Universidade Regional de Blumenau - FURB. \\ termus@ibest.com.br
}

RESUMO

As melhores práticas de governança corporativa prevêem que a gestão da empresa conheça os riscos assumidos e que os investidores sejam informados sobre eles. Este trabalho tem como objetivo levantar as práticas de divulgação sobre riscos adotadas nas Informações Anuais (IANs) das empresas. Apresenta-se uma análise do conteúdo desses instrumentos de comunicação das empresas classificadas no nível de governança do Novo Mercado da Bovespa. 
A metodologia da pesquisa aplicada caracteriza-se como descritiva e qualitativa, empregando como método a análise documental. Os resultados mostram que 73 das 99 empresas do Novo Mercado haviam divulgado suas IANs até 31.05.08, sendo que 12 delas nada relatam sobre suas políticas de gestão de riscos e das 61 restantes apenas 6 apresentam um item específico sobre gestão de risco em seus reportes. A análise de conteúdo mostra que as empresas que adotam as melhores práticas oferecem diversas informações sobre gestão de risco, incluindo estrutura organizacional, técnicas utilizadas e políticas de proteção. Conclui-se que os reportes sobre riscos da maioria das empresas ainda apresentam níveis baixos de evidenciação.

\section{Palavras-chave: Novo Mercado. Gestão de Risco. Governança Corporativa.}

\section{ABSTRACT}

Organizations from different industries are exposed to market, operational, credit, legal and other risks. This work aims to analyze the information about risk management presented in the IAN (annual information) offered to CVM by the Brazilian companies listed in the segment of the Bovespa New Market index. The methodology of the applied research is characterized as descriptive, applying qualitative research and documentary analysis. The results show that 73 of the 99 Brazilian public companies participants in Bovespa's New Market index, had disclosed the IAN about the year 2007 by May 31, 2008, and from these 73 companies, 12 reported nothing about their risk policies in the reports studied. The article presents an analysis of the 61 remaining companies identifying the companies that devote a specific topic of their reports to risk management and the information presented on these topics. It follows that the annual information of the Brazilian public companies listed in Bovespa's New Market index has relatively low levels of disclosure on risk management.

Key words: Bovespa's New Market. Risk management. Corporate governance.

\section{RESUMEN}

Las mejores prácticas de gobierno corporativo previenen que la gestión de la empresa conozca los riesgos asumidos y que los inversionistas sean informados sobre ellos. Este trabajo tiene como objetivo levantar las prácticas de divulgación sobre riesgos adoptadas en las Informaciones Anuales (IANs) de las empresas. Se presenta un análisis del contenido de estos instrumentos de comunicación de las empresas clasificadas en el nivel de gobierno del Nuevo Mercado de la Bovespa. La metodología de la pesquisa aplicada se caracteriza como descriptiva y cualitativa, empleando como método el análisis documental. Los resultados muestran que 73 de las 99 empresas del Nuevo Mercado habían 
divulgado sus IANs hasta 31.05.08, siendo que 12 de ellas nada relatan sobre su políticas de gestión de riesgos y de las 61 restantes apenas 6 presentan un apartado especifico sobre gestión de riesgo en sus informes. El análisis de contenido muestra que las empresas que adoptan las mejores prácticas ofrecen diversas informaciones sobre gestión de riesgo, incluyendo estructura organizacional, técnicas utilizadas y políticas de protección. Se concluye que los reportes sobre riesgos de la mayoría de las empresas aún presentan niveles bajos de evidencia.

Palabras-clave: Nuevo Mercado. Gestión de Riesgo. Gobierno Corporativo.

\section{INTRODUÇÃO}

As novas pressões de mercado resultantes da atual crise econômica e os escândalos financeiros ocorridos nos últimos anos motivaram os bancos centrais de países desenvolvidos a desenvolverem instrumentos para oferecer maior estabilidade e credibilidade ao sistema financeiro internacional. Exemplos desses instrumentos são os acordos da Basiléia I e II. Nesta mesma linha de pensamento, Pereira (2006, p.105) apresenta seu posicionamento sobre o tema, afirmando que todas as empresas, financeiras ou não, são submetidas a diversos tipos de riscos, que podem ser internos (endógenos) ou externos (exógenos) às atividades da entidade. Segundo o autor, "denomina-se análise do risco o estudo dos eventos que têm efeitos sobre a atividade da empresa", e quanto à administração de riscos, Pereira ainda conceitua que "é aplicação de estratégias para reduzir os custos gerados pelos riscos".

Pinho Lopes e Moreno (2004), ao apresentarem suas considerações sobre riscos, destacam que fazer distinção entre volatilidade, incerteza e risco é necessário para serem iniciadas as abordagens sobre o tema. Dessa forma, os autores referem que, havendo inconstância, mudança de visão com facilidade, há a caracterização de volatilidade; já ao abordar incerteza, referem os autores que, ao se estudar determinada decisão, as possibilidades e probabilidades de ocorrência associadas são conhecidas, mas a sua determinação com exatidão não é possível, se tem caracterizada a incerteza; por fim, risco no entendimento dos autores é probabilidade de determinado evento ou fato acontecer sem a vontade dos interessados.

O Instituto Brasileiro de Governança Corporativa (IBGC) define risco como qualquer relação com a vida pessoal ou empresarial que envolva possibilidade de perda ou oportunidade de ganhos, apontando que, para o campo financeiro, investimentos carregam riscos inerentes ao retorno esperado para esse investimento depende do aumento do valor da empresa (IBGC, 2007). Divulgou um manual sobre Gerenciamento de Riscos Corporativos, referenciando que o Conselho de Administração de uma entidade deve de forma antecipada, assegurar-se que a direção da empresa possui ferramentas (sistemas) 
de identificação de a quais riscos o negócio em questão está exposto, quais são as possibilidades desses riscos ocorrerem e medidas para reduzir a possibilidade da ocorrência ao longo das atividades da entidade (IBGC, 2007).

Duarte Júnior et al (2001), em estudo sobre controles internos no contexto de gestão de riscos, refere as seguintes expressões sobre gerenciamento de riscos, mas com enfoque em instituições financeiras, afirmando que formação e criação de cultura interna para gerenciar as exposições de riscos a que a entidade está exposta; assim, a linguagem relativamente à qual a entidade irá coordenar seus esforços para mitigar os riscos será uniforme, para as diversas áreas da empresa, criando dessa maneira nomenclatura única, que para os autores é ponto facilitador para gerenciar a exposição aos riscos e criar cultura de gestão de riscos.

Dentro do contexto referenciado denota-se que empreender significa buscar um retorno econômico-financeiro adequado ao nível de risco associado à atividade. Ou seja, o risco é inerente à atividade de negócios, na qual a consciência do risco e a capacidade de administrá-lo, aliadas à disposição de correr riscos e de tomar decisões, são elementos-chaves. Assumir riscos diferencia empresas líderes, mas também pode levá-las a estrondosos fracassos. O resultado das iniciativas de negócios revela que o risco pode ser gerenciado a fim de subsidiar os administradores na tomada de decisão, visando a alcançar objetivos e metas dentro do prazo, do custo e das condições pré-estabelecidas (IBGC, 2007).

Este estudo propõe a seguinte questão problema: Como se podem comparar as práticas de divulgação de riscos das empresas que aderem ao nível de governança do Novo Mercado da Bovespa? Assim, este artigo terá como proposição analisar as informações sobre gestão de riscos nas Informações Anuais das empresas (IANs), estabelecendo uma comparação entre elas.

Para responder à questão problema da pesquisa, o artigo tem a seguinte estrutura: introdução; referencial teórico apresentando considerações sobre a Gestão de Riscos; apresentação breve sobre Governança Corporativa e os Níveis de Governança; metodologia da pesquisa; estudo das empresas classificadas; e consideração finais.

\section{REVISÃO BIBLIOGRÁFICA}

Bernstein (apud ZANQUETA FILHO e LUGON, 1997, p. 8) afirma que:

Ao longo dos tempos o homem vem aprendendo, continuamente, a conviver com o risco. Para isso vem desenvolvendo inúmeros métodos para seu gerenciamento.A fronteira entre os tempos modernos e o passado está na capacidade de domínio do risco, considerando-se que o futuro não seria somente um capricho dos deuses e que a humanidade não estaria eternamente à mercê dos fenômenos da natureza. Desde que haja a possibilidade de desconhecer do futuro, cada ser humano é um gerente de risco, não por escolha mas por absoluta necessidade de sobrevivência. 
Ao analisar conceitos de risco, Lemgruber et al (2001) referem que há quatro tipos distintos de risco nas empresas, quais sejam: risco de mercado, risco operacional, risco de crédito e risco legal. Para os autores, esses tipos de risco estão relacionados diretamente com qualquer operação do mercado financeiro.

Os riscos, com a ênfase com que estão sendo estudados agora, tem sua importância salientada em virtude dos fatos ocorridos com o Barings Bank, Procter\&Gamble, Bankers Trust, Gibson Greentings, Orange Country, Metallgensellschaft, entre outros, conforme se posicionam Lemgruber et al (2001).

Seguindo essa linha de entendimento, o objetivo geral deste artigo é o de analisar o conteúdo dos relatórios Informações Anuais (IANs) emitidos pelas empresas listadas no Nível Novo Mercado de Governança Corporativa da Bovespa em maio de 2008, relatórios referentes ao período de 2007 quanto à divulgação sobre as práticas de gestão de riscos. Optou-se por analisar apenas as empresas listadas no Novo Mercado porque tais empresas têm um nível maior de exigência em relação à evidenciação de suas informações, o que decorre dos padrões de Governança Corporativa exigidos pela Bovespa.

Para Jorion (2003, p. 333), a tendência ao reforço no gerenciamento global do risco é motivada por dois fatores: "a exposição às novas fontes de risco e a maior volatilidade de novos produtos". Considera ainda o autor que, com a ampla negociação de títulos financeiros ao redor do mundo, criaram-se novas exposições a riscos, como, por exemplo, as exposições às perdas provocadas pelas alterações nas taxas de câmbio das moedas em diferentes locais do mundo.

\subsection{Gestão de Riscos}

A gestão de riscos tem recebido crescente atenção por parte dos gestores nos últimos anos. Um exemplo importante disso é a importância dada aos riscos de mercado, que passaram de um simples componente da gestão de carteiras de investimento para uma ferramenta essencial na gestão de resultados e fluxo de caixa, não somente de instituições financeiras, mas para qualquer empresa cujas atividades estejam submetidas às flutuações de preços, sobretudo as que operam negócios para os quais haja disponibilidade de instrumentos financeiros que possam ser utilizados como hedge ou proteção. Corroborando essa idéia, Malleta e Coelho (2004) afirmam que o risco de mercado tornou-se, ao longo dos últimos anos, uma das maiores preocupações para os participantes do mercado financeiro; agências reguladoras, bancos comerciais e de investimentos, grandes corporações e investidores estão cada vez mais focando suas atenções ao nível de risco ao qual sua instituição ou carteira de investimentos estão submetidos.

Duarte Júnior (2005), por sua vez, aponta, ao introduzir a gestão de riscos, as quatro dimensões do risco (tipos de risco), sendo que sua descrição se aproxima da de Lemgruber 


\section{repc}

et al (2001), ao afirmar que os riscos a que as empresas estão expostas podem ser classificados em risco de mercado, risco operacional, risco de crédito e risco legal.

Crisante Neto e Cresto (2003) iniciam suas considerações sobre como controlar e minimizar os efeitos dos riscos nas entidades empresariais apontando que as variáveis tempo, prazo e preço são importantes para controlar perdas ou os riscos de possíveis perdas; e definem, de forma contundente, que controlar essas variáveis é tão importante quanto a empresa apresentar ao mercado novos produtos.

Crouhy, Galai e Mark (2004, p.1), analisando a importância da gestão de risco nas empresas, afirmam que "[...] se há preocupação quanto à capacidade da instituição de gerenciar o risco, então o preço de suas ações será penalizado [...]".

Ao apresentar suas considerações sobre métodos de cálculo de risco, Lemgruber et al (2001, p. 105) destacam que "[...] em comum, as metodologias para estimação do risco requerem conhecimentos sobre a mecânica dos mercados de interesse, alguma sofisticação matemática e sistemas computacionais e de informações confiáveis". Posto isso, abordam as peculiaridades da mensuração de cada tipo de risco de forma breve, apontando que "[...] no caso de risco operacional e risco legal, o problema de medir risco deve ser tratado em uma abordagem caso por caso. Tratando-se de risco de mercado e risco de crédito, algumas metodologias já se encontram em uso, e explicativas na literatura de finanças".

Como o escopo deste estudo é o de identificar quais as empresas listadas no NM da Bovespa que informam práticas de gestão de riscos, é importante que sejam apresentados os diferentes métodos de medição de riscos, muito embora o propósito aqui não seja o de aprofundar seus conceitos e técnicas.

Há vários métodos disponíveis na literatura para que seja dimensionado o valor dos riscos aos quais estão expostas as empresas.

Perobelli (2004) destaca que métodos utilizados em instituições financeiras estão sendo adaptados para empresas não financeiras, face aos objetivos diferentes dos tipos de instituições citadas. Perobelli (2004) também destaca documento de 1999 - Corporate Metrics Technical Document, emitido pelo Risk Metrics Group -, que trata do estudo e da apresentação das técnicas do Earnings at Risk - EaR (Ganhos em Risco), Earnings-per-Share-at-Risk - EPSaR, e CFaR (Cash Flow at Risk), todos variações do VaR - Value at Risk (Valor em Risco).

O VaR, para Crouhy, Galai e Mark (2004), pode ser definido como a pior perda que poderia ser esperada em decorrência de se deter um título ou uma carteira por um dado período de tempo, dado um nível especificado de confiança estatística.

\subsection{Controle de gestão e avaliação de riscos}

Sobre a necessidade de controle de gestão Gomes e Salas (2001, p. 21) referem que: 
o interesse sobre controle de gestão em aumentado nos últimos anos, em decorrência principalmente das rápidas mudanças ocorridas no contexto social e organizacional, a partir de 1973, com a crise mundial do petróleo. Grande parte das empresas passou a desenvolver-se em um contexto social e organizacional caracterizado por grande instabilidade, muito complexo e bastante hostil que passou a exigir um constante aperfeiçoamento dos sistemas de controle com vistas a enfrentar uma concorrência acirrada, decorrente a globalização da economia.

Adicionam ainda os autores que as instabilidades freqüentes e a conseqüente adequação dos sistemas de controle foram mais sentidas nas empresas que tinham resultados estáveis, fazendo com que estas criassem formas formais de adequação à nova realidade. Posto isso, os autores referem que a Contabilidade de Gestão tradicional está baseada nas seguintes características (GOMES; SALAS, 2001):
a) o processo de Controle tem ênfase no resultado e é medido por indicadores quantitativos;
b) o departamento de controle financeiro assume papel de destaque no proces- so administrativo - coleta / processa e manipula as informações contábeis e de orçamento;
c) no processo de controle, há uma clara separação das etapas desse processo (planejamento, informação e avaliação);
d) as relações de avaliação de investimento são basicamente econômico financeiras;
e) os sistemas de informação das empresas são integrados e centralizados na Contabilidade, e tem caráter econômico-financeiro;
f) o processo de avaliação é basicamente qualitativo, e de curto prazo;
g) os incentivos estão resumidos em salários, comissões, participações nos lucros;
h) este sistema complementa-se, na prática, pela supervisão direta realizada pela gerência por uma disciplina rígida;

Contrapondo a concepção tradicional da Contabilidade, Gomes e Salas (2001) destacam aspectos da sociedade após a década de 1980, quais sejam:
a) a mudança tecnológica é constante, melhorando posições estratégicas das empresas;
b) há percepção flagrante de hábitos e posicionamento de instituições sociais tradicionais;
c) ocorreu desestatização de inúmeros países, ou seja, o estado deixou de ser influente em setores da economia, que agora tem a ampla e livre concorrência como situação cotidiana;
d) movimentos econômicos para regiões onde até então não havia relevância monetária;


Lowenkron (apud Dotti e Silva, 2004) apresenta seu posicionamento sobre a gestão de riscos no contexto de proteção do valor da empresa, relacionando os seguintes benefícios de se adotarem práticas de gestão de riscos:
a) aumento da transparência;
b) melhoria na comunicação entre as unidades de negócio;
c) auxílio na decisão de hedge;
d) avaliação de impacto de cenários adversos no desempenho da empresa;
e) implantação de um processo de cultura de gestão de riscos;
f) impedimento de perdas indesejadas e desastres financeiros.

Em específico, quanto à alavancagem do valor da firma, os benefícios são:
a) identificação de projetos menos arriscados (através do CashFlow-at-Risk);
b) melhoria do rating e conseqüente redução da taxa de captação;
c) redução dos gastos com impostos pelo do Earnings-at-Risk.

\subsection{Riscos, mercado de capitais brasileiro e governança corporativa na Bovespa}

No Brasil não há ainda, por parte da CVM, a obrigatoriedade da divulgação dos tipos de riscos a que as empresas estão expostas. Pode ocorrer que outros órgãos, como o Banco Central do Brasil, por exemplo, obriguem as empresas a divulgarem de alguma maneira os riscos a que estão expostas. Até o mês de outubro de 2008, a única norma relacionada ao assunto era a Instrução CVM n. ${ }^{\circ} 235 / 95$, que se refere à obrigatoriedade pelas companhias abertas de evidenciar em notas explicativas o valor de mercado de seus derivativos financeiros, estejam eles reconhecidos ou não em suas demonstrações financeiras. Recentemente foi editada a Deliberação CVM 550, de 17.10.2008, que dispõe sobre a apresentação de informações sobre instrumentos financeiros derivativos em nota explicativa às informações trimestrais (ITR). Embora não seja ainda uma evidenciação que contenha a avaliação dos riscos por parte da empresa, pelo menos institui a obrigatoriedade de que sejam declarados todos os ativos e passivos que contenham riscos de mercado.

A CVM obriga também as empresas listadas na bolsa de valores a divulgarem o formulário IAN (Informações Anuais). Conforme a Instrução Normativa n. ${ }^{\circ}$ 202/93 e suas alterações, esse formulário contém informações corporativas, operacionais, societárias, entre outras. Tal documento deve ser apresentado à CVM e à Bolsa de Valores. O IAN deve ser visto como um documento dinâmico sobre a evolução dos negócios da empresa, pois é uma das formas objetivas de comunicação da empresa para o mercado.

Em dezembro de 2000 a Bovespa lançou o novo mercado e os níveis diferenciados de governança corporativa, caracterizando segmentos especiais de valores de mercado. Conforme Beuren, Gallon e Hein (2006), o novo mercado e os níveis diferenciados de governança possuem 
regras de listagem diferenciadas, destinadas à negociação de ações emitidas por empresas que se comprometem, voluntariamente, com a adoção de práticas de governança corporativa e com a prestação de informações adicionais e de vanguarda em relação ao que é exigido pela legislação. Para o IBGC - Instituto Brasileiro de Governança Corporativa, a governança corporativa pode ser considerada como um conjunto de práticas e relacionamentos entre os acionistas ou cotistas, conselho de administração, diretoria, auditoria independente e conselho fiscal, com a finalidade de otimizar o desempenho da empresa e facilitar o acesso ao capital. Em 31.05.2008, data do fechamento desta pesquisa, havia 99 empresas listadas no Novo Mercado, constituindo-se na população do presente estudo.

\section{METODOLOGIA DA PESQUISA}

Para a consecução dos objetivos, foi utilizada a pesquisa descritiva, sendo o trabalho desenvolvido com as fases de identificar, relatar e comparar dados. Conforme Gil (2007, p. 42), a pesquisa descritiva tem como objetivo primordial a descrição das características de determinada população ou fenômeno ou o estabelecimento de relações entre variáveis. Uma de suas características mais significativas está na utilização de técnicas padronizadas de coleta de dados.

Na busca por respostas ao problema de pesquisa e sua abordagem, o trabalho está inserido também no enfoque da pesquisa qualitativa, que freqüentemente é aplicada no desenvolvimento de pesquisas descritivas nas quais procura-se descobrir e classificar a relação entre variáveis, assim como na investigação da relação de causalidade entre os fenômenos, de causa e efeito.

Quanto aos procedimentos para este trabalho, foi utilizada a pesquisa documental e a análise de conteúdo, que, segundo Bardin (2004; 131), "é um conjunto de técnicas de análise das comunicações". Sobre o instrumento de comunicação no qual se baseia uma pesquisa documental, Bardin (2004, p. 31) afirma que "com maior rigor, será um único instrumento, mas marcado por uma grande disparidade de formas e adaptável a um campo de atuação muito vasto: as comunicações".

O estudo definiu como forma de comunicação das empresas a ser analisada as IANs, e nestas aplicou a técnica da análise de conteúdo para alcançar formas de responder à pergunta deste trabalho.

Bauer e Gaskell (2002, p. 212) apontam como vantagens da análise de conteúdo os seguintes fatores: "[...] é sistemática e pública; ela faz uso principalmente de dados brutos que ocorrem naturalmente; pode lidar com grandes quantidades de dados; presta-se para dados históricos; [...]".

Para Bardin (2004, p. 43), o objetivo da análise de conteúdo pode ser assim apresentado:

Um conjunto de técnicas de análise das comunicações visando obter, por procedimentos sistemáticos e objetivos de descrição do conteúdo das mensagens, indicadores (quantitativos ou não) que permitam a inferência de conhecimentos relativos às condições de produção / recepção (variáveis inferidas) destas mensagens. 
Bardin (2004) sustenta que a análise de conteúdo usa a análise de documentos para efetivar seu objetivo, ou seja, estudar documentos para de outra forma comunicar ou apresentar informações de acordo com a conveniência do estudo, que por ora a utiliza. A autora refere que esse procedimento pode se fazer valer das seguintes formas de análise de documentos: "os resumos ou abstracts", ou a "indexação, que permite, por classificação em palavras-chave, descritores ou índices, classificar os elementos de informação dos documentos $[\ldots] "$.

\section{ANÁLISE DOS RESULTADOS}

Na Tabela 1, apresentam-se as empresas listadas no Novo Mercado da Bovespa na data de fechamento desta pesquisa (31/05/2008). A coluna "Situação IAN", informa: D - divulgou a IAN, N.D - não havia divulgado a IAN até a data de fechamento deste estudo.

\section{Tabela 1 - Empresas Pesquisadas}

\begin{tabular}{|c|c|c|c|}
\hline Nr. & Razão Social & Nome de Pregão & Situação IAN \\
\hline 1 & Abyara Planejamento Imobiliário S.A. & & \\
\hline ABYARA & D. & & \\
\hline 2 & Açúcar Guarani S.A. & & \\
\hline GUARANI & N.D. & & \\
\hline 3 & Agra Empreendimentos Imobiliários S.A. & & \\
\hline AGRA INCORP & D. & & \\
\hline 4 & American Banknote S.A. & ABNOTE & D. \\
\hline 5 & Amil Participações S.A. & & \\
\hline AMIL & N.D. & & \\
\hline 6 & B2W - Companhia Global Do Varejo & B2W VAREJO & N.D. \\
\hline 7 & Bco Brasil S.A. & BRASIL & D. \\
\hline 8 & Bco Nossa Caixa S.A. & NOSSA CAIXA & N.D. \\
\hline 9 & Bematech S.A. & BEMATECH & D. \\
\hline 10 & $\begin{array}{c}\text { Bolsa De Mercadorias e Futuros - } \\
\text { BMF S.A. }\end{array}$ & & \\
\hline BMF & D. & & \\
\hline 11 & Bovespa Holding S.A. & BOVESPA HLD & N.D. \\
\hline 12 & Br Malls Participações S.A. & & \\
\hline BR MALLS PAR & D. & & \\
\hline 13 & Brascan Residential Properties S.A. & BRASCAN RES & N.D. \\
\hline 14 & Brasil Brokers Participações S.A. & & \\
\hline BR BROKERS & D. & & \\
\hline 15 & Brasil Ecodiesel Ind Com Bio.OI.Veg.S.A. & ECODIESEL & D. \\
\hline 16 & Brasilagro - Cia Bras De Prop Agrícolas & & \\
\hline
\end{tabular}




\begin{tabular}{|c|c|c|c|}
\hline Nr. & Razão Social & Nome de Pregão & Situação IAN \\
\hline BRASILAGRO & D. & & \\
\hline 17 & Camargo Correa Desenv. Imobiliário S.A. & CC DES IMOB & D. \\
\hline 18 & Cia Concessões Rodoviárias & CCR RODOVIAS & D. \\
\hline 19 & Cia Hering & CIA HERING & N.D. \\
\hline 20 & Cia Providência Indústria e Comércio & & \\
\hline PROVIDENCIA & D. & & \\
\hline 21 & Cia Saneamento Básico Est Sao Paulo & & \\
\hline SABESP & N.D. & & \\
\hline 22 & $\begin{array}{c}\text { Cia Saneamento De Minas Gerais-Copasa } \\
\text { MG }\end{array}$ & COPASA & D. \\
\hline 23 & Company S.A. & COMPANY & N.D. \\
\hline 24 & Construtora Tenda S.A. & TENDA & D. \\
\hline 25 & Cosan S.A. Indústria e Comércio & & \\
\hline COSAN & D. & & \\
\hline 26 & Cpfl Energia S.A. & CPFL ENERGIA & D. \\
\hline 27 & Cr2 Empreendimentos Imobiliários S.A. & CR2 & N.D. \\
\hline 28 & Cremer S.A. & CREMER & D. \\
\hline 29 & Csu Cardsystem S.A. & CSU CARDSYST & N.D. \\
\hline 30 & Cyrela Brazil Realty S.A.Empreend e Part & CYRELA REALT & D. \\
\hline 31 & Cyrela Commercial Propert S.A. Empr Part & CYRE COM-CCP & D. \\
\hline 32 & Datasul S.A. & DATASUL & D. \\
\hline 33 & Diagnósticos da América S.A. & & \\
\hline DASA & N.D. & & \\
\hline 34 & Drogasil S.A. & DROGASIL & D. \\
\hline 35 & Edp - Energias Do Brasil S.A. & ENERGIAS BR & D. \\
\hline 36 & Embraer-Empresa Brás. de Aeronáutica S.A. & & \\
\hline EMBRAER & N.D. & & \\
\hline 37 & Equatorial Energia S.A. & EQUATORIAL & D. \\
\hline 38 & Eternit S.A. & ETERNIT & D. \\
\hline 39 & Even Construtora e Incorporadora S.A. & & \\
\hline EVEN & $\mathrm{D}$. & & \\
\hline 40 & Ez Tec Empreend. e Participações S.A. & & \\
\hline EZTEC & D. & & \\
\hline 41 & Fertilizantes Heringer S.A. & FER HERINGER & D. \\
\hline 42 & Gafisa S.A. & GAFISA & D. \\
\hline 43 & General Shopping Brasil S.A. & GENERALSHOPP & N.D. \\
\hline 44 & Grendene S.A. & GRENDENE & D. \\
\hline 45 & Gvt (Holding) S.A. & GVT HOLDING & D. \\
\hline 46 & Helbor Empreendimentos S.A. & HELBOR & D. \\
\hline 47 & Hypermarcas S.A. & HYPERMARCAS & D. \\
\hline 48 & Ideiasnet S.A. & IDEIASNET & D. \\
\hline 49 & Iguatemi Empresa De Shopping Centers S.A & IGUATEMI & D. \\
\hline
\end{tabular}




\begin{tabular}{|c|c|c|c|}
\hline Nr. & Razão Social & Nome de Pregão & Situação IAN \\
\hline 50 & Indústrias Romi S.A. & INDS ROMI & D. \\
\hline 51 & Inpar S.A. & INPAR S/A & N.D. \\
\hline 52 & Invest Tur Brasil - Desenv. Imob. Tur.S.A. & INVEST TUR & D. \\
\hline 53 & lochpe Maxion S.A. & IOCHP-MAXION & D. \\
\hline 54 & JBS S.A. & JBS & D. \\
\hline 55 & JHSF Participações S.A. & JHSF PART & D. \\
\hline 56 & Klabin Segall S.A. & KLABINSEGALL & D. \\
\hline 57 & Le Lis Blanc Deux Com Confec Roupas S.A. & LE LIS BLANC & D. \\
\hline 58 & Light S.A. & LIGHT S/A & N.D. \\
\hline 59 & Localiza Rent A Car S.A. & LOCALIZA & N.D. \\
\hline 60 & Log-In Logística Intermodal S.A. & LOG-IN & N.D. \\
\hline 61 & Lojas Renner S.A. & LOJAS RENNER & D. \\
\hline 62 & LPS Brasil - Consultoria De Imóveis S.A. & LOPES BRASIL & N.D. \\
\hline 63 & Lupatech S.A. & LUPATECH & N.D. \\
\hline 64 & M.Dias Branco S.A. Ind Com De Alimentos & M.DIASBRANCO & D. \\
\hline 65 & Magnesita Refratários S.A. & MAGNESITA SA & D. \\
\hline 66 & Marfrig Frigorificos E Com De Alim S.A. & MARFRIG & D. \\
\hline 67 & Marisa S.A. & MARISA & N.D. \\
\hline 68 & Medial Saúde S.A. & MEDIAL SAUDE & D. \\
\hline 69 & Metalfrio Solutions S.A. & METALFRIO & D. \\
\hline 70 & Minerva S.A. & MINERVA & D. \\
\hline 71 & MMX Mineração e Metálicos S.A. & MMX MINER & D. \\
\hline 72 & MPX Energia S.A. & MPX ENERGIA & D. \\
\hline 73 & MRV Engenharia e Participações S.A. & MRV & D. \\
\hline 74 & Natura Cosméticos S.A. & NATURA & D. \\
\hline 75 & Obrascon Huarte Lain Brasil S.A. & OHL BRASIL & D. \\
\hline 76 & Odontoprev S.A. & ODONTOPREV & D. \\
\hline 77 & Pdg Realty S.A. Empreend. e Participações & PDG REALT & D. \\
\hline 78 & Perdigão S.A. & PERDIGAO S/A & N.D. \\
\hline 79 & Porto Seguro S.A. & PORTO SEGURO & D. \\
\hline 80 & Portobello S.A. & PORTOBELLO & N.D. \\
\hline 81 & Positivo Informática S.A. & POSITIVO INF & D. \\
\hline 82 & Profarma Distrib. Prod. Farmacêuticos S.A. & PROFARMA & D. \\
\hline 83 & Redecard S.A. & REDECARD & D. \\
\hline 84 & Renar Macas S.A. & RENAR & N.D. \\
\hline 85 & Rodobens Negócios Imobiliários S.A. & RODOBENSIMOB & D. \\
\hline 86 & Rossi Residencial S.A. & ROSSI RESID & D. \\
\hline 87 & São Carlos Empreend. e Participações S.A. & SAO CARLOS & D. \\
\hline 88 & São Martinho S.A. & SAO MARTINHO & D. \\
\hline 89 & Satipel Industrial S.A. & SATIPEL & D. \\
\hline 90 & SLC Agrícola S.A. & SLC AGRICOLA & D. \\
\hline 91 & Springs Global Participações S.A. & SPRINGS & N.D. \\
\hline
\end{tabular}




\begin{tabular}{|l|c|c|c|}
\hline Nr. & Razão Social & Nome de Pregão & Situação IAN \\
\hline 92 & Tecnisa S.A. & TECNISA & D. \\
\hline 93 & Tegma Gestão Logística S.A. & TEGMA & D. \\
\hline 94 & Tempo Participações S.A. & TEMPO PART & N.D. \\
\hline 95 & Totvs S.A. & TOTVS & D. \\
\hline 96 & TPI - Triunfo Particip. e Invest. S.A. & TRIUNFO PART & D. \\
\hline 97 & Tractebel Energia S.A. & TRACTEBEL & D. \\
\hline 98 & Trisul S.A. & TRISUL & D. \\
\hline 99 & Weg S.A. & WEG & D. \\
\hline
\end{tabular}

Fonte: Dados da pesquisa

Das 99 empresas que se encontravam listadas no Novo Mercado da Bovespa, foram excluídas 26 empresas que não tinham divulgado as IANs do período de 2007 até a data de fechamento da pesquisa. Assim, restaram $73,73 \%$ do total das empresas listadas no Novo Mercado, havendo entre elas empresas do mais variados ramos de atividades.

Verifica-se que na Tabela 2, as 12 empresas destacadas que nada relataram sobre riscos nos formulários da IAN do exercício 2007 disponibilizados.

\section{Tabela 2 - Empresas que não apresentam informações sobre riscos nas IANs}

\begin{tabular}{|l|l|l|}
\hline Nr. & Razão Social & Nome de Pregão \\
\hline 1 & American Banknote S.A. & ABNOTE \\
\hline 2 & Brasilagro - Cia Bras. de Prod. Agrícolas & BRASILAGRO \\
\hline 3 & Cia Providência Indústria e Comércio & PROVIDENCIA \\
\hline 4 & Cyrela Brazil Realty S.A. Empreend e Part & CYRELA REALT \\
\hline 5 & Drogasil S.A. & DROGASIL \\
\hline 6 & Fertilizantes Heringer S.A. & FER HERINGER \\
\hline 7 & Idéiasnet S.A. & IDEIASNET \\
\hline 8 & Lojas Renner S.A. & LOJAS RENNER \\
\hline 9 & Magnesita Refratários S.A. & MAGNESITAAS \\
\hline 10 & MMX Mineração e Metálicos S.A. & MMX MINER \\
\hline 11 & Obrascon Huarte Lain Brasil S.A. & OHL BRASIL \\
\hline 12 & Porto Seguro S.A. & PORTO SEGURO \\
\hline
\end{tabular}

Fonte: Dados da pesquisa

As empresas da Tabela 2 representam 16,44\% das 73 empresas pesquisadas. Essas 12 organizações não divulgam informações sobre os riscos a que estão expostas e nem apresentam algum tópico específico sobre como estão tratando esses riscos nas suas organizações, conforme os relatórios de informações anuais do período. 
$\mathrm{Na}$ Tabela 3, que analisa as informações anuais das 61 empresas restantes, vê-se que 41 delas, ou seja, 67,21\% apresentam tópico específico sobre riscos nas suas IANs, abordando a forma com que estão tratando e gerenciando este assunto. As demais 20 empresas, ou 32,79\%, não apresentam tópico referente a risco, mas apenas tratam o assunto de forma generalista, sem informar de forma específica os cuidados adotados em seus processos de gestão dos riscos a que estão expostas.

\section{Tabela 3 - Tópico específico sobre risco nas IANs}

\begin{tabular}{|c|c|c|}
\hline Nr. & Razão Social & Tópico Específico sobre Risco \\
\hline 1 & Abyara Planejamento Imobiliário S.A. & Não \\
\hline 2 & Agra Empreendimentos Imobiliários S.A. & Sim \\
\hline 3 & Bco Brasil S.A. & Sim \\
\hline 4 & Bematech S.A. & Não \\
\hline 5 & Bolsa de Mercadorias e Futuros-Bmf S.A. & $\operatorname{Sim}$ \\
\hline 6 & Br Malls Participações S.A. & Sim \\
\hline 7 & Brasil Brokers Participações S.A. & Não \\
\hline 8 & Brasil Ecodiesel Ind. Com. Bio.OI.Veg. S.A. & Não \\
\hline 9 & Camargo Correa Desenv. Imobiliário S.A. & Sim \\
\hline 10 & Cia Concessões Rodoviárias & Não \\
\hline 11 & Cia Saneamento de Minas Gerais-Copasa MG & $\operatorname{Sim}$ \\
\hline 12 & Cosan S.A. Indústria e Comércio & Sim \\
\hline 13 & Construtora Tenda S.A. & Sim \\
\hline 14 & Cremer S.A. & Não \\
\hline 15 & CPFL Energia S.A. & Sim \\
\hline 16 & Cyrela Commercial Propert S.A. Empr. Part. & Sim \\
\hline 17 & Datasul S.A. & Sim \\
\hline 18 & Edp - Energias do Brasil S.A. & $\operatorname{Sim}$ \\
\hline 19 & Equatorial Energia S.A. & Sim \\
\hline 20 & Eternit S.A. & Não \\
\hline 21 & Even Construtora e Incorporadora S.A. & Sim \\
\hline 22 & Ez Tec Empreend. e Participações S.A. & $\operatorname{Sim}$ \\
\hline 23 & Gafisa S.A. & $\operatorname{Sim}$ \\
\hline 24 & Grendene S.A. & $\operatorname{Sim}$ \\
\hline 25 & GVT (Holding) S.A. & Sim \\
\hline 26 & Helbor Empreendimentos S.A. & Sim \\
\hline 27 & Hypermarcas S.A. & Sim \\
\hline 28 & Iguatemi Empresa de Shopping Centers S.A. & Não \\
\hline 29 & Indústrias Romi S.A. & Não \\
\hline 30 & Invest. Tur. Brasil - Desenv. Imob. Tur. S.A. & $\operatorname{Sim}$ \\
\hline 31 & lochpe Maxion S.A. & Sim \\
\hline 32 & JBS S.A. & Não \\
\hline
\end{tabular}




\begin{tabular}{|c|c|c|}
\hline 33 & Jhsf Participações S.A. & Sim \\
\hline 34 & Klabin Segall S.A. & Não \\
\hline 35 & Le Lis Blanc Deux Com. Confec. Roupas S.A. & Sim \\
\hline 36 & M. Dias Branco S.A. Ind. Com. de Alim. S.A. & Sim \\
\hline 37 & Marfrig Frigoríficos e Com. de Alim. S.A. & Sim \\
\hline 38 & Medial Saúde S.A. & Não \\
\hline 39 & Metalfrio Solutions S.A. & Sim \\
\hline 40 & Minerva S.A. & Sim \\
\hline 41 & Mpx Energia S.A. & Sim \\
\hline 42 & Mrv Engenharia e Participações S.A. & Sim \\
\hline 43 & Natura Cosméticos S.A. & Não \\
\hline 44 & Odontoprev S.A. & Não \\
\hline 45 & Pdg Realty S.A. Empreend. e Participações & Sim \\
\hline 46 & Positivo Informática S.A. & Sim \\
\hline 47 & Profarma Distrib. Prod. Farmacêuticos S.A. & Não \\
\hline 48 & Redecard S.A. & Sim \\
\hline 49 & Rodobens Negócios Imobiliários S.A. & Sim \\
\hline 50 & Rossi Residencial S.A. & Sim \\
\hline 51 & São Carlos Empreend. e Participações S.A. & Sim \\
\hline 52 & São Martinho S.A. & Não \\
\hline 53 & Satipel Industrial S.A. & Sim \\
\hline 54 & SLC Agrícola S.A. & Sim \\
\hline 55 & Tecnisa S.A. & Sim \\
\hline 56 & Tegma Gestão Logística S.A. & Não \\
\hline 57 & Totvs S.A. & Não \\
\hline 58 & TPI - Triunfo Particip. e Invest. S.A. & Sim \\
\hline 59 & Tractebel Energia S.A. & Sim \\
\hline 60 & Trisul S.A. & Não \\
\hline 61 & Weg S.A. & Não \\
\hline
\end{tabular}

Fonte: Dados da pesquisa

Dentre as organizações que incluem um tópico sobre riscos nas IANs (Tab. 3), destaca-se que as empresas lochpe Maxion S.A. e Datasul S.A., apesar de incluírem um tópico específico sobre risco em seu relatório, tratam-nos de forma generalista, não oferecendo nenhum detalhamento sobre os riscos a que a empresa está exposta.

Destaca-se no outro extremo o Banco do Brasil, que possui tópico especial sobre risco. Ressalte-se que, sendo uma instituição financeira, a empresa segue as determinações do Banco Central do Brasil, o que inclui o respeito à obrigatoriedade de divulgar suas políticas de risco. A Copasa, além do tópico específico, mostra uma tabela do impacto dos riscos nas taxas de juros e variação cambial, assim como a Minerva S.A., que divulga que elimina seus riscos de variação cambial realizando operações de hedge. As demais organizações 
não-financeiras divulgam de forma espontânea informações sobre os riscos a que estão expostas, dando maior transparência em seus relatórios para os usuários das informações.

Tabela 4 - Método de avaliação dos riscos

\begin{tabular}{|l|l|c|c|}
\hline Nr. & Razão Social & Declara o Método & Qual Método \\
\hline 1 & Bco. Brasil S.A. & Sim & VaR \\
\hline 2 & $\begin{array}{l}\text { Bolsa de Mercadorias e Futuros - BMF } \\
\text { S.A. }\end{array}$ & Sim & EVT \\
\hline 3 & SLC Agrícola S.A. & Sim & CFaR \\
\hline
\end{tabular}

Fonte: Dados da pesquisa.

$\mathrm{Na}$ Tabela 4 foram destacadas as organizações que informam que medem os riscos a que estão expostas e qual o método utilizado na avaliação de riscos como definido por Lowenkron (apud DOTTI e SILVA, 2004). Das 61 empresas da amostra, 3 delas, ou 4,92\%, declaram o método de avaliação dos riscos. Analisando de outra forma, essas 3 empresas representam $7,31 \%$ das 41 empresas que possuem item específico sobre riscos no seu relatório anual de informações de 2007. Os métodos declarados são: Value et Risk (VaR) no Banco do Brasil; o Extreme Value Thory, (EVT) na Bolsa de Mercadorias e Futuros - BMF S.A.; e o Cash Flow at Risk (CFaR), que é adotado na empresa SLC Agrícola S.A., onde predomina a atividade do setor primário da economia. Como menciona a literatura, o VaR é utilizado na maioria para instituições financeiras, já para as não-financeiras o CFaR atende melhor às expectativas dos gestores na área de risco. Outras organizações como Helbor, Lê Lis Blanc Deux, Minerva e São Carlos Empreendimentos não divulgam o método em si, mas apresentam um item específico sobre Avaliação Quantitativa e Qualitativa dos Riscos do Mercado.

Tabela 5 - Existência de tópico específico sobre outros aspectos de gestão de risco

\begin{tabular}{|l|l|l|}
\hline r. & Razão Social & Nome de Pregão \\
\hline 1 & Bco. Brasil S.A. & BRASIL \\
\hline 2 & Bolsa de Mercadorias e Futuros - BMF S.A. & BMF \\
\hline 3 & Datasul S.A. & DATASUL \\
\hline 4 & Edp - Energias do Brasil S.A. & ENERGIAS BR \\
\hline 5 & Equatorial Energia S.A. & EQUATORIAL \\
\hline 6 & Tractebel Energia S.A. & TRACTEBEL \\
\hline
\end{tabular}

Fonte: Dados da pesquisa

As organizações listadas na Tabela 5, conforme as IANs de 2007, apresentam um item específico sobre outros aspectos da gestão de riscos, incluindo informações sobre unidades 
administrativas, como comitês ou departamentos, específicas para o gerenciamento dos riscos a que estão expostas. Note-se que são apenas 6 empresas de um total de 61, representando $9,84 \%$ das empresas que apresentaram um tópico de gestão de riscos em suas IANs. Destacase que a empresa SLC Agrícola, mesmo tendo declarado o método pelo qual avalia os seus riscos, não apresentou em suas IANs nenhum tópico específico sobre outros aspectos da gestão de risco. Já as empresas Datasul, Energias do Brasil, Equatorial Energia e Tractebel apresentam informações sobre outros aspectos da gestão de riscos, mas não declaram o método com que os avaliam. A empresa Natura Cosméticos declara que existem quatro comitês na empresa e um deles se caracteriza como comitê de gestão de riscos, porém não trouxe esta informação num tópico específico como evidenciado nas demais organizações.

\section{CONCLUSÕES}

Este estudo apresentou uma análise de conteúdo das Informações Anuais (IANs) do ano de 2007, prestadas pelas 99 empresas participantes do Novo Mercado da Bovespa, tendo sido analisadas as 61 empresas desse grupo que haviam divulgado até 31 de maio de 2008 as referidas IANs. Constatou-se que 41 empresas apresentam um item específico sobre risco nos relatórios, oferecendo informações mais detalhadas sobre os riscos a que estão expostas. Uma exceção deve ser feita para as empresas lochpe Maxion e DATASUL, que, mesmo tendo tópico específico sobre riscos em seus relatórios, tratam-nos de forma generalista.

Apesar de a literatura mencionada neste trabalho atribuir grande importância ao gerenciamento de riscos, apenas 3 das 61 empresas listadas na pesquisa, ou 4,92\%, declaram os métodos de avaliação de risco que utilizam. Essas empresas são Banco do Brasil, que utiliza como método de avaliação o VaR; a BMF, que adota o Extreme Value Thory (EVT); e SLC Agrícola, que utiliza o método de avaliação de Cash Flow at Risk (CFaR) ou Fluxo de Caixa em Risco. Das empresas que apresentam um tópico específico de gestão de risco em suas IANs, apenas 6 , representando 9,84\%, declaram possuir um setor específico para a coordenação da gestão de riscos em sua estrutura organizacional.

Conclui-se que as Informações Anuais das companhias abertas brasileiras apresentam níveis relativamente baixos de evidenciação sobre gestão de riscos nas organizações. Apesar dos avanços trazidos pela Deliberação CVM 550, de 17.10.2008, as evidências trazidas pela atual crise econômica demonstram que as informações sobre a gestão de risco merecem destaque muito maior do que o verificado, sobretudo quando se considera que as empresas pesquisadas são as que, ao aderirem ao Novo Mercado da Bovespa, inserem-se no grupo das que adotam os melhores padrões de governança corporativa do mercado brasileiro. As evidências colhidas pela pesquisa permitem inferir que num futuro próximo as demonstrações contábeis e as informações anuais da CVM deverão sujeitar-se a um processo de profunda melhoria em termos de evidenciação da exposição a riscos existente nas empresas. 


\section{REFERÊNCIAS}

CRISANTE Neto, Beraldo; CRESTO, Vicente. Risco operacional: o porquê de se registrarem perdas. Resenha BM\&F. São Paulo: Bolsa de Mercadorias \& Futuros, n.156, jul./ago. 2003.

CROUHY, Michael; GALAI, Dan; MARK, Robert. Gerenciamento de risco: uma abordagem conceitual e prática: uma visão integrada dos riscos de crédito, operacional e de mercado. Traduzido por Carlos H. Trieschmann e Luiz Frazão Filho. Rio de Janeiro: Qualitymark; São Paulo: Serasa, 2004.

BARDIN, L. Análise de conteúdo. 3. ed. Tradução de Luís Antero Reto e Augusto Pinheiro. São Paulo: Edições 70, 2004.

BEUREN, Ilse Maria (Org.). Como elaborar trabalhos monográficos em contabilidade: teoria e prática. 3. ed. São Paulo: Atlas, 2006,

BEUREN, Ilse M.; GALLON, Alexandra V.; HEIN, Nelson. Itens Obrigatórios e de Divulgação Espontânea no Relatório de Administração. CONGRESSO DE CONTABILIDADE, Coimbra, 2006. Anais... Coimbra/Portugal: ISCAC, 2006. CD- ROM.

BOVESPA. Bolsa de Valores de São Paulo: Novo Mercado Empresas Listadas. Disponível em: www.bovespa.com.br. Acesso em: 26 maio 2008.

BRASIL. Deliberação n. ${ }^{\circ} 550$ da Comissão de Valores Mobiliários, de 17 de outubro de 2008.

DOTTI, Gabriela; SILVA; César Augusto Tibúrcio. Risco operacional: uma relação empírica com o valor de mercado das empresas de telecomunicações. In: $4^{\circ}$ CONGRESSO USP DE CONTROLADORIA E CONTABILIDADE, 4., São Paulo, 2004. Anais... São Paulo: FEA/USP, 2004.

DUARTE JÚNIOR, Antonio Marcos. A importância do gerenciamento de riscos corporativos. Disponível em: <www.risktech.com.br/PDFs/RISCORPO.pdf>. Acesso em: 26 maio 2008.

DUARTE JR, Antonio Marcos. Risco: definições, tipos, medição e recomendações para seu gerenciamento. In: Lemgruber; E.C. et al (Org.). Gestão de riscos e derivativos: aplicação no Brasil. São Paulo: Atlas, 2001.

GIL, Antonio Carlos. Como elaborar projetos de pesquisa. 4. ed. 10 Reimpressão. São Paulo: Atlas, 2007. 


\section{repc}

GOMES, Josir Simeone; SALAS, J.M.A. Controle de gestão: uma abordagem contextual e organizacional. São Paulo: Atlas, 1997.

GUIMARÃES, Terence Augusto (Coord.). Melhores práticas na gestão do risco operacional grupo de trabalho melhores práticas. Disponível em <www.febraban.org.br/p5 a_52gt34++5cv8_4466+ff145afbb52ffrtg33fe36455li5411pp+e/sitefebraban/GTMP_documento_finalago2006.pdf>. Acesso em: 26 maio 2008.

INSTITUTO BRASILEIRO DE GOVERNANÇA CORPORATIVA. Guia de orientação para o gerenciamento de riscos corporativos. Coordenação: Eduarda La Rocque. São Paulo, SP: IBGC, 2007. Disponível em: <www.ibgc.gov.br>. Acesso em: 26 maio 2008.

INSTITUTO BRASILEIRO DE GOVERNANÇA CORPORATIVA. Código das Melhores Praticas de Governança Corporativa. Organização IBGC. São Paulo, SP: IBGC. Disponível em: <www.ibgc.gov.br>. Acesso em: 26 maio 2008.

JORION, P., Value-at-Risk: A nova fonte de referência para a gestão do risco financeiro. São Paulo: BM\&F, 2003.

LA ROCQUE, Eduarda de; LOWENKRON, Alexandre. Métricas e particularidades da gestão de risco em corporações. Disponível em: <www.riskcontrol.com.br/arquivos/Artigos/Métricas\%20e\%20Particularidades.pdf>. Acesso em: 10 abr. 2008.

LOPES, Wander de Pinho; MORENO, Roberto. Uma reflexão sobre taxonomia de risco, seus índices e aspectos qualitativos, na avaliação de investimentos estrangeiros diretos. EnANPAD, 28., 2004, Curitiba. Anais ... Rio de Janeiro: ANPAD, 2004.

LOWENKRON, Alexandre. Potencialização do valor da firma através do risk management corporativo. Disponível em: <www.riskcontrol.com.br>. Acesso em: 10 abr. 2008.

PEREIRA, José Matias. Gestão do risco operacional: uma avaliação do novo acordo de capitais

- Basiléia II. Revista Contemporânea de Contabilidade. Ano 03, Vol. 1, n. 6, jul./dez. 2006.

PEROBELLI, Fernanda Finotti Cordeiro; et al. Testando diferentes métodos de estimação do cash flow at risk TD. Mestrado (dissertação) - Economia Aplicada FEA/ UFJF, Juiz de Fora, 2007. 
MALETTA, Bruno Vasques; COELHO, Fábio Henrique de Sousa. Modelos baseados em simulação de Monte Carlo: soluções para o cálculo do value-at-risk. In: Congresso EnANPAD, 28., 2004, Curitiba. Anais... Rio de Janeiro: ANPAD, 2004.CD-ROM.

SILVA, César Augusto Tibúrcio; MUNHOZ, Django Agrahyde. A utilização do lucro contábil como proxy do risco no Brasil. ENANPAD, 30., 2006, Salvador. Anais... Rio de Janeiro: ANPAD, 2006.

ZANQUETTO Filho, Hélio; LUGON, Valmir B. Pereira. Modelo para gestão do risco nas propostas de seguro de automóvel, com base no perfil sócio-econômico e cultural do segurado, utilizando análise discriminante. ENANPAD, 28., 2004, Curitiba. Anais... Rio de Janeiro: ANPAD, 2004. 